\title{
Bok regulates mitochondrial fusion and morphology
}

\author{
Jacqualyn J. Schulman ${ }^{1} \cdot$ Laura M. Szczesniak ${ }^{1}{ }^{1} \cdot$ Eric N. Bunker ${ }^{2} \cdot$ Heather A. Nelson ${ }^{3} \cdot$ Michael W. Roe $^{4}$. \\ Larry E. Wagner $I^{5} \cdot$ David I. Yule ${ }^{5} \cdot$ Richard J. H. Wojcikiewicz $^{1}$
}

Received: 7 November 2018 / Revised: 5 March 2019 / Accepted: 25 March 2019 / Published online: 11 April 2019

(c) ADMC Associazione Differenziamento e Morte Cellulare 2019

\begin{abstract}
Bok (Bcl-2-related ovarian killer) is a member of the Bcl-2 protein family that governs the intrinsic apoptosis pathway, but the cellular role that Bok plays is controversial. Remarkably, endogenous Bok is constitutively bound to inositol 1,4,5trisphosphate receptors $\left(\mathrm{IP}_{3} \mathrm{Rs}\right)$ and is stabilized by this interaction. Here we report that despite the strong association with $\mathrm{IP}_{3} \mathrm{Rs}$, deletion of Bok expression by CRISPR/Cas9 (clustered regularly interspaced short palindromic repeats/CRISPRassociated protein-9 nuclease)-mediated gene editing does not alter calcium mobilization via $\mathrm{IP}_{3} \mathrm{Rs}$ or calcium influx into the mitochondria. Rather, Bok deletion significantly reduces mitochondrial fusion rate, resulting in mitochondrial fragmentation. This phenotype is reversed by exogenous wild-type Bok and by an $\mathrm{IP}_{3} \mathrm{R}$ binding-deficient Bok mutant, and may result from a decrease in mitochondrial motility. Bok deletion also enhances mitochondrial spare respiratory capacity and membrane potential. Finally, Bok does not play a major role in apoptotic signaling, since Bok deletion does not alter responsiveness to various apoptotic stimuli. Overall, despite binding to $\mathrm{IP}_{3} \mathrm{Rs}$, Bok does not alter $\mathrm{IP}_{3} \mathrm{R}$-mediated $\mathrm{Ca}^{2+}$ signaling, but is required to maintain normal mitochondrial fusion, morphology, and bioenergetics.
\end{abstract}

\section{Introduction}

Bok (Bcl-2-related ovarian killer) is a member of the Bcl-2 protein family that governs the intrinsic apoptosis pathway [1-3]. These proteins contain conserved Bcl-2 homology (BH) domains (BH1-4) and the family is made up of antiapoptotic proteins (e.g., Bcl-2 and Mcl-1), pro-apoptotic

Edited by L. Scorrano

Richard J. H. Wojcikiewicz

Wojcikir@upstate.edu

1 Department of Pharmacology, SUNY Upstate Medical University, 750 E Adams Street, Syracuse, NY 13210, USA

2 Biochemistry Section, Surgical Neurology Branch, National Institute of Neurological Disorders and Stroke, National Institutes of Health, Bethesda, MD, USA

3 Department of Cell and Developmental Biology, SUNY Upstate Medical University, Syracuse, NY, USA

4 Department of Cell and Developmental Biology, Department of Medicine, SUNY Upstate Medical University, Syracuse, NY, USA

5 Department of Pharmacology and Physiology, University of Rochester, Rochester, NY, USA proteins (e.g., Bak and Bax), and BH3-only proteins (e.g., Bid and Bad). A complex network of interactions between these family members controls apoptosis and cell viability [1-3]. Bok has been generally grouped with Bak and Bax on the basis of high sequence homology and initial in vitro studies showing that overexpressed Bok induced apoptosis in cultured cells [4-6]. Endogenous Bok is predominately localized to the endoplasmic reticulum (ER) [7], whereas many Bcl-2 family members predominately localize to the mitochondrial membrane [1,8]. Although Bok is ubiquitously expressed, Bok-knockout (KO) mice are phenotypically normal [4].

Currently, the role that Bok plays in apoptosis is unclear, with various studies showing both pro- and anti-apoptotic characteristics. On the one hand, overexpressed exogenous Bok can promote apoptosis [7, 9-11], purified Bok can permeabilize the outer membrane of isolated mitochondria and artificial liposomes [9, 11, 12], and Bok-KO mouse embryonic fibroblasts (MEFs) exhibit reduced susceptibility to ER stress-induced apoptosis [13, 14]. On the other hand, Bok-KO MEFs have also been shown to exhibit increased susceptibility to ER stress-induced apoptosis [7, 15], and Bok is neuroprotective during $\mathrm{Ca}^{2+}$-induced neuronal injury, by suppressing poly ADP-ribose polymerase (PARP)-dependent cell death [16]. Additionally, evidence has been presented that Bok plays no role in apoptosis, 
since Bok-KO neurons, hematopoietic cells, and nonneuronal cells have all been shown to respond normally to several apoptotic stimuli $[4,7,16]$.

Uniquely for the Bcl-2 protein family, Bok binds very strongly to inositol 1,4,5-trisphosphate $\left(\mathrm{IP}_{3}\right)$ receptors $\left(\mathrm{IP}_{3} \mathrm{Rs}\right)$ and, indeed, in unperturbed cells all Bok appears to be $\mathrm{IP}_{3} \mathrm{R}$ bound $[17,18]$. $\mathrm{IP}_{3} \mathrm{Rs}$ are $\sim 300 \mathrm{kDa}$ proteins that form tetrameric, $\mathrm{IP}_{3^{-}}$and $\mathrm{Ca}^{2+}$-gated $\mathrm{Ca}^{2+}$ channels in the ER membranes of mammalian cells and play an essential role in intracellular $\mathrm{Ca}^{2+}$ signaling [19, 20]. There are three highly homologous $\mathrm{IP}_{3} \mathrm{R}$ forms $\left(\mathrm{IP}_{3} \mathrm{R} 1-3\right)$ that can form both homo- and heterotetramers $[19,20]$. Binding of $\mathrm{IP}_{3}$ and $\mathrm{Ca}^{2+}$ to $\mathrm{IP}_{3} \mathrm{Rs}$ induces a yet-to-be-defined conformational change in the tetrameric channel that allows $\mathrm{Ca}^{2+}$ to flow from stores within the ER lumen into the cytosol to increase cytosolic $\mathrm{Ca}^{2+}$ concentration $[19,20]$. In addition, the $\mathrm{Ca}^{2+}$ released by $\mathrm{IP}_{3} \mathrm{Rs}$ can be taken up by adjacent mitochondria where it plays a role in regulating ATP production $[21,22]$. $\mathrm{IP}_{3} \mathrm{Rs}$ also play a role in intrinsic apoptosis, and various effects of Bcl-2 family members on $\mathrm{IP}_{3} \mathrm{R}$ activity and $\mathrm{ER} \mathrm{Ca}^{2+}$ content have been reported [22-24]. Bok binds strongly to only $\mathrm{IP}_{3} \mathrm{R} 1$ and $\mathrm{IP}_{3} \mathrm{R} 2$, with the binding mediated by the $\mathrm{BH} 4$ domain of Bok and a small region within the $\mathrm{IP}_{3} \mathrm{R}$ coupling domain at approximately amino acid $~ 1900$ [17, 18]. The interaction of Bok with $\mathrm{IP}_{3} \mathrm{Rs}$ protects them from proteolysis either by chymotrypsin in vitro or by caspase-3 in vivo, apparently by steric hindrance of protease access [17, 18]. In addition, Bok is dramatically stabilized by binding to $\mathrm{IP}_{3} \mathrm{Rs}$ and unbound Bok is ubiquitinated and degraded by the proteasome [17].

While Bcl-2 family members are well known for their ability to influence apoptosis, they also regulate mitochondrial dynamics (e.g., fusion and fission), morphology, and function [25, 26]. Mitochondrial fusion and fission allow for growth, the exchange of mitochondrial content, the elimination of damaged mitochondria, and the balance between fusion and fission controls overall mitochondrial morphology [25, 27]. Bcl-2 family members, such as Bax, Bak, Bcl$\mathrm{xl}$, and Mcl-1, have been shown to regulate mitochondrial dynamics through interactions with either mitofusin 2 (Mfn2) (a dynamin-like GTPase that regulates fusion of the outer mitochondrial membrane) or dynamin-related protein 1 (Drp-1) (a dynamin-like GTPase that regulates mitochondrial fission) [28-34]. Thus, the network of interactions between Bcl-2 family members also likely govern mitochondrial morphology [25, 26, 30].

Here we report that despite the strong interaction between Bok and $\mathrm{IP}_{3} \mathrm{Rs}$, Bok deletion does not alter $\mathrm{IP}_{3} \mathrm{R}$ mediated $\mathrm{Ca}^{2+}$ mobilization or $\mathrm{Ca}^{2+}$ influx into the mitochondria. Rather, Bok deletion results in mitochondrial fragmentation, due to a decrease in mitochondrial fusion rate, and an enhancement of mitochondrial spare respiratory capacity and membrane potential. Lastly, Bok deletion does not alter responsiveness to several apoptotic stimuli. These findings provides new insight into the cellular role of Bok.

\section{Materials and methods}

\section{Materials}

MEFs, $\alpha$ T3, and HEK 293 T cells were cultured as described $[35,36]$. DT40 cells were maintained at $39^{\circ} \mathrm{C}$ and $5 \% \mathrm{CO}_{2}$ in RPMI-1640 supplemented with $1 \%$ chicken serum, $10 \%$ fetal bovine serum, $10 \mu \mathrm{M} \beta$-mercaptoethanol, $100 \mathrm{U} / \mathrm{mL}$ penicillin, and $100 \mu \mathrm{g} / \mathrm{mL}$ streptomycin. Antibodies raised in rabbits were: anti-IP ${ }_{3} \mathrm{R} 1$ and anti- $\mathrm{IP}_{3} \mathrm{R} 2$ [35], anti-erlin2 [37], anti-IP ${ }_{3} \mathrm{R} 1-3$ [38], anti-Mcl-1 \#D35A5, anti-Bcl-xL \#54H6, anti-Bcl-2 \#50E3, anti-caspase-3 \#9662, anti-PARP \#9542, anti-Mfn2 \#9482, anti-VDAC1 \#4661, and antipDrp1-616 \#3455 (Cell Signaling Technology), anti-Bak \#06-536 (Millipore), anti-Bax \#N-20 and anti-Tom20 \#FL145 (Santa Cruz Biotechnology Inc.), anti-Bok, raised against amino acids 19-32 of mouse Bok [4, 7], and antiMiro1 \#HPA010687 (Atlas Antibodies). Mouse monoclonal antibodies were: anti-FLAG epitope clone M2 (Sigma), anti-IP ${ }_{3} \mathrm{R} 3$, raised against amino acids 22-230 of human $\mathrm{IP}_{3} \mathrm{R} 3$ (BD Transduction Labs), anti-Mfn1 \#D-10 (Santa Cruz Biotechnology Inc.), anti-Drp-1 \#14647 (Cell Signaling Technology), anti-electron transport chain complexes \#110413 (Abcam), and anti-p97 (Research Diagnostics Inc.). Horseradish peroxidase-conjugated secondary antibodies, gonadotropin-releasing hormone (GnRH), oleoyl-L- $\alpha$-lysophosphatidic acid (LPA), protease inhibitors, Triton X-100, 3-[(3-cholamidopropyl)-dimethylammonio]1-propanesulfonate (CHAPS), brefeldin A (BFA), tunicamycin (TN), and thapsigargin (TG) were purchased from Sigma. Dithiothreitol, Precision Plus ${ }^{\mathrm{TM}}$ Protein Standards, and sodium dodecyl sulfate-polyacrylamide gel electrophoresis (SDS-PAGE) reagents were from Bio-Rad. Protein A-Sepharose CL-4B was from GE Healthcare. MG132 was from Biomol. Staurosporine (SST) was from Enzo Life Sciences. Linear, MW 25,000 polyethylenimine was from Polysciences Inc. MitoTracker ${ }^{\mathrm{TM}}$ Red and Deep Red, tetramethylrhodamine ethyl ester perchlorate (TMRE), and Lipofectamine LTX were from Thermo Fisher.

\section{Cell lysis, immunoprecipitation, SDS-PAGE, crude mitochondrial fractionation, and mass spectrometry}

To prepare cell lysates for SDS-PAGE or for immunoprecipitation (IP), cells were harvested with ice-cold lysis buffer (150 mM NaCl, $50 \mathrm{mM}$ Tris-HCl, $1 \mathrm{mM}$ EDTA, 1\% CHAPS, or $1 \%$ Triton $\mathrm{X}-100,10 \mu \mathrm{M}$ pepstatin, $0.2 \mathrm{mM}$ phenylmethylsulfonyl fluoride, $1 \mathrm{mM}$ dithiothreitol, and 0.2 $\mu \mathrm{M}$ soybean trypsin inhibitor, $\mathrm{pH} 8.0$ ). CHAPS was used 
for all experiments involving IP. Lysates were incubated on ice for $30 \mathrm{~min}$ and clarified by centrifugation at $16,000 \times g$ for $10 \mathrm{~min}$ at $4{ }^{\circ} \mathrm{C}$. To IP-specific proteins, clarified lysates were incubated with antisera and protein A-Sepharose CL$4 \mathrm{~B}$ for $\sim 16 \mathrm{~h}$ at $4{ }^{\circ} \mathrm{C}$, and IPs were washed thoroughly with lysis buffer. To obtain crude mitochondrial fractions, cells were harvested with ice-cold mitochondrial isolation buffer (MIB) (20 mM HEPES, $220 \mathrm{mM}$ mannitol, $70 \mathrm{mM}$ sucrose, $1 \mathrm{mM}$ EGTA, $2 \mathrm{mg} / \mathrm{mL}$ bovine serum albumin (BSA), and $0.5 \mathrm{mM}$ phenylmethylsulfonyl fluoride, $\mathrm{pH} 7.6$ ), incubated on ice for $5 \mathrm{~min}$, and homogenized with 40 strokes of a drill-fitted pestle. Homogenates were centrifuged at $800 \times g$ for $5 \mathrm{~min}$ at $4{ }^{\circ} \mathrm{C}$, supernatants were centrifuged at $10,000 \times$ $g$ for $20 \mathrm{~min}$ at $4{ }^{\circ} \mathrm{C}$, pellets were resuspended in MIB without BSA, were centrifuged at $10,000 \times g$ for $10 \mathrm{~min}$ at $4{ }^{\circ} \mathrm{C}$, and finally pellets were resuspended in MIB without BSA. All samples were resuspended in gel-loading buffer, incubated at $37{ }^{\circ} \mathrm{C}$ for $30 \mathrm{~min}$, subjected to SDS-PAGE, and proteins were transferred to nitrocellulose for probing as described [17]. Immunoreactivity was detected using Pierce ECL reagents and a Genegnome imager (Syngene Bio Imaging) or a Chemidoc (Bio-Rad). For mass spectrometry, Coomassie blue-stained $\mathrm{IP}_{3} \mathrm{R} 1$ and Bok bands were cut from SDS-PAGE gels, were destained, combined, trypsinized, and analyzed by liquid chromatography with tandem mass spectrometry, using a LTQ Orbitrap XL ${ }^{\mathrm{TM}}$ Hybrid Ion Trap-Orbitrap mass spectrometer (Thermo Fisher).

\section{Generation and analysis of Bok-KO MEF, aT3, and DT40 cell lines}

The CRISPR/Cas9 system [39, 40] was used to target exons within the Bok gene. Oligonucleotides that contained the target sequences (MEFs and $\alpha \mathrm{T} 3$, exon 2: ACGCGCGGC TTTTGCGCGC, or exon 4: CCCCGCGGCCACGGAA TAC; DT40, exon 2: GAGCTTGTGTCCCAAGCCA, or exon 4: GCCATGGTCCACACCATCG) were annealed and ligated into AflII-linearized guide RNA (gRNA) vector (AddGene). MEFs, $\alpha \mathrm{T} 3$, and DT40 cells were NEON transfected with Bok-gRNA constructs and vectors encoding hCas9 (Addgene) and enhanced green fluorescent protein (EGFP) (Clontech). EGFP-expressing cells were selected 48 $\mathrm{h}$ later by fluorescence-activated cell sorting and plating at 1 cell/well in a 96-well plate. Colonies were expanded and screened in immunoblots for Bok immunoreactivity, which yielded Bok-KO cell lines. Multiple independent cell lines from each exon target were used for all experiments.

\section{Generation and analysis of reconstituted MEF cell lines}

Stable expression of exogenous Bok was obtained by NEON transfection of Bok-KO MEF cell lines with 3F-
WTBok or 3F-L34GBok complementary DNAs [17], followed by selection in $0.6 \mathrm{mg} / \mathrm{mL}$ G418 for $72 \mathrm{~h}$, and plating at $1 \mathrm{cell} /$ well in a 96-well plate. Colonies were expanded and screened in immunoblots for FLAG immunoreactivity, which yielded Bok-KO ${ }^{+\mathrm{WTBOK}}$ and Bok-KO ${ }^{+\mathrm{L} 34 \mathrm{GBOK}}$ cell lines that were maintained in $0.3 \mathrm{mg} / \mathrm{mL} \mathrm{G} 418$.

\section{Bok depletion}

Bok depletion was achieved using a Mission short hairpin RNA (shRNA) pLKO.1 plasmid (Sigma-Aldrich) expressing shRNA targeting mouse Bok (5'-CCGGAGCTTCT TGCTTAGGCTATTTCTCGAGAAATAGCCTAAGCAA GAAGCTTTTTTG-3'). This plasmid and a non-targeting shRNA control (SHC002) were transiently co-transfected with pRSV-REV (Addgene plasmid \#12253), pMDL/pRRE (Addgene plasmid \#12251), and pCMV-VSVG (Addgene plasmid \#8454) into HEK $293 \mathrm{~T}$ cells using polyethylenimine as described [17]. Media were changed $24 \mathrm{~h}$ later, virus-containing supernatants were collected 48 and $72 \mathrm{~h}$ later, combined, filtered through a $0.45 \mu \mathrm{m}$ filter (Corning), and stored at $-80{ }^{\circ} \mathrm{C}$ until used to transduce MEFs in the presence of $9 \mu \mathrm{g} / \mathrm{mL}$ polybrene, followed by selection with $2 \mu \mathrm{g} / \mathrm{mL}$ puromycin for $72 \mathrm{~h}$.

\section{Cytoplasmic $\mathrm{Ca}^{2+}$ measurements}

Cytosolic-free $\mathrm{Ca}^{2+}$ concentration $\left(\left[\mathrm{Ca}^{2+}\right]_{\mathrm{c}}\right)$ in cell suspensions was measured as described [18], except that cells were loaded with $1 \mu \mathrm{M}$ Fura 2-AM for $1 \mathrm{~h}$ at $37^{\circ} \mathrm{C}$ and were washed three times with a Krebs/HEPES buffer. $\left[\mathrm{Ca}^{2+}\right]_{c}$ was calculated using the Grynkiewicz equation [41]. For single-cell $\left[\mathrm{Ca}^{2+}\right]_{\mathrm{c}}$ measurements, cells were plated on glass coverslips, incubated for $15 \mathrm{~min}$ at $37^{\circ} \mathrm{C}$ with $1 \mu \mathrm{M}$ Fura 2$\mathrm{AM}, 0.01 \%$ (vol/ $/ \mathrm{vol})$ pluronic acid in a standard extracellular solution $\left(135 \mathrm{mM} \mathrm{NaCl}, 6 \mathrm{mM} \mathrm{KCl}, 1 \mathrm{mM} \mathrm{CaCl}_{2}\right.$, $1 \mathrm{mM} \mathrm{MgCl} 2,10 \mathrm{mM}$ HEPES-NaOH, $5.5 \mathrm{mM}$ D-glucose, and $0.2 \%$ BSA, pH 7.4), then placed into a temperaturecontrolled microperifusion chamber mounted on an inverted fluorescence spinning disk confocal microscope (Olympus IX81), and continuously superfused with standard extracellular solution $(2 \mathrm{~mL} / \mathrm{min})$ at $37^{\circ} \mathrm{C}$. Cells were visualized with a $\times 40$ oil-immersion objective. Fura 2 excitation wavelengths were 340 and $380 \mathrm{~nm}$ with emission measured at $510 \mathrm{~nm}$. Data are expressed as the fold-change in $340 /$ $380 \mathrm{~nm}$ ratio normalized to resting, unstimulated values.

\section{Mitochondrial $\mathrm{Ca}^{2+}$ measurements}

To measure mitochondrial-free $\mathrm{Ca}^{2+}$ concentration $\left(\left[\mathrm{Ca}^{2+}\right]_{\mathrm{m}}\right)$, MEFs were transfected with a vector encoding $2 \mathrm{mtD} 3 \mathrm{cpv}$, a fluorescence resonance energy transfer (FRET)-based indicator localized to the mitochondrial matrix [42], using the 
NEON Transfection System (Invitrogen; $10 \mu \mathrm{g}$ total DNA per $100 \mu \mathrm{L}$ cell suspension at $3 \times 10^{7}$ cells $/ \mathrm{mL}, 1$ pulse, $20 \mathrm{~ms}$, $1500 \mathrm{~V})$, and $48 \mathrm{~h}$ later cells were plated on glass coverslips. Single-cell $\left[\mathrm{Ca}^{2+}\right]_{\mathrm{m}}$ was measured as described for single-cell $\left[\mathrm{Ca}^{2+}\right]_{\mathrm{c}}$ measurements, but $2 \mathrm{mtD} 3 \mathrm{cpv}$ was excited at $440 \mathrm{~nm}$ and emission was measured at $485 \mathrm{~nm}$ (FRET donor) and $535 \mathrm{~nm}$ (FRET acceptor). Data are expressed as the foldchange in $535 / 485 \mathrm{~nm}$ ratio normalized to resting, unstimulated values.

\section{Single $I P_{3} R 1$ channel measurements}

Preparation of DT40 cell nuclei, on-nuclear patch clamp, and data analysis was performed as described [43]. Data shown are from exon 4-targeted DT40-3KO $+\mathrm{rIP}_{3} \mathrm{R} 1$ cells, but essentially identical data were obtained from exon 2targeted DT40-3KO $+\mathrm{rIP}_{3} \mathrm{R} 1$ cells.

\section{Live cell fluorescence microscopy of mitochondrial morphology}

MEFs were NEON transfected with a plasmid encoding DsRed2-Mito (Clontech), and $48 \mathrm{~h}$ later, cells were set onto 1.5 glass cover dishes (Cellvis). Cells were imaged with a Nikon structured illumination microscope (N-SIM, Ti microscope) equipped with a $\times 100$ SR Apo TIRF oilimmersion objective, an electron multiplying chargedcoupled device camera (iXon Em-CCD, Andor), using 561 $\mathrm{nm}$ excitation/605 $\mathrm{nm}$ emission. Z-stacks of entire cells were taken, followed by reconstruction of the threedimensional z-stacks and deconvolution using NISElements software (Nikon). For additional confocal analysis, MEFs were either NEON transfected with a plasmid encoding DsRed2-Mito or stained with MitoTracker Red as per the manufacturer's instructions, and cells were imaged with a Nikon $\mathrm{C} 2$ microscope (Ti microscope) equipped with a $\times 60$ Plan Apo VC oil-immersion objective lens, using $543.5 \mathrm{~nm}$ excitation $/ 585 \mathrm{~nm}$ emission. To measure mitochondrial particle velocity, DsRed2-Mito-expressing MEFs were continuously imaged for 5 min and velocity of mitochondrial particles that could be tracked for at least 30 $\mathrm{s}$ was measured using the Tracking module in NISElements software. All microscopy was done at $37^{\circ} \mathrm{C}$ with $5 \% \mathrm{CO}_{2}$. All images were subjected to one iteration of blind deconvolution and mitochondrial particle quantification was done using NIS-Elements software.

\section{Mitochondrial fusion assay}

MEFs were transiently transfected using Lipofectamine LTX as per the manufacturer's instructions to express photoactivatable GFP targeted to the mitochondrial matrix (mito-PAGFP) [30]. After $48 \mathrm{~h}, \mathrm{MEFs}$ were stained with
MitoTracker Deep Red as per the manufacturer's instructions, and cells were imaged with a Zeiss 880 Airy microscope equipped with a Plan-Apochromat $\times 63 / 1.40$ oil objective. A small region of interest was photoactivated with a $405 \mathrm{~nm}$ laser and $\mathrm{z}$-stacks of the entire cells were acquired at 0 and $30 \mathrm{~min}$ post-photoactivation. GFP was imaged using $488 \mathrm{~nm}$ excitation/516 nm emission and MitoTracker Deep Red was imaged using $633 \mathrm{~nm}$ excitation/654 nm emission. A custom MATLAB script was used to determine the fraction of mitochondria positive for GFP. Briefly, images were background subtracted, PAGFPpositive cells were manually selected, and mitochondria were defined on the basis of size and an automated intensity threshold for each z-stack. Mitochondria were deemed GFP-positive if GFP intensity was above a GFP intensity threshold for each cell, defined as the mean plus two times the SD of pixel intensities before photoactivation. The fraction of mitochondria positive for GFP was then calculated for 0 and $30 \mathrm{~min}$ after photoactivation to determine the fraction of mitochondrial particles above the GFP intensity threshold.

\section{Measurement of oxygen consumption rate}

MEFs were seeded in XFe96 plates (Agilent) at 40,000 cells/well, and $24 \mathrm{~h}$ later, oxygen consumption rate (OCR) was measured using the Seahorse XFe96 Extracellular Flux Analyzer and the XF Cell Mito Stress Test Kit (Agilent), as per the manufacturer's instructions. Cells were washed three times with unbuffered XF media supplemented with $10 \mathrm{mM}$ D-glucose, $2 \mathrm{mM}$ L-glutamine, and $1 \mathrm{mM}$ pyruvate, $\mathrm{pH} 7.4$, were incubated at $37^{\circ} \mathrm{C}$ in a $\mathrm{CO}_{2}$-free environment for $1 \mathrm{~h}$, and triplicate measurements of baseline followed by triplicate measurements after the addition of each drug $(1 \mu \mathrm{M}$ oligomycin, $1 \mu \mathrm{M}$ carbonyl cyanide- $p$-trifluoromethoxyphenyl-hydrazone (FCCP), and $0.5 \mu \mathrm{M}$ rotenone/antimycin A, respectively) were collected. Afterwards, a cell normalization assay was performed (cyQUANT, Thermo Fisher), as per the manufacturer's instructions and measured with a Synergy H1 microplate reader and Gen 5 software (BioTek) using $485 \mathrm{~nm}$ excitation/528 $\mathrm{nm}$ emission. OCR measurements were normalized to the cell densities.

\section{Measurement of mitochondrial membrane potential}

MEFs were seeded at $1.0 \times 10^{6}$ cells $/ 9.6 \mathrm{~cm}^{2}$ well, incubated with $500 \mathrm{nM}$ TMRE for $20 \mathrm{~min}$ at $37^{\circ} \mathrm{C}$ and $5 \% \mathrm{CO}_{2}$, rinsed and harvested with $10 \mathrm{mM}$ HEPES, $154 \mathrm{mM} \mathrm{NaCl}, 1$ $\mathrm{mM}$ EDTA, $\mathrm{pH} 7.4$, centrifuged at $3000 \times g$ for $1 \mathrm{~min}$, resuspended with phosphate-buffered saline and $2 \%$ fetal bovine serum, and analyzed by flow cytometry using a LSR II Cell Analyzer (Becton Dickinson). For all samples, 10,000 cell events were recorded and data were analyzed 
using the Flow Jo software to determine the geometric mean for TMRE fluorescence.

\section{Data analysis}

All experiments were repeated two or more times $(N=$ the number of independent experiments) and representative images of gels and traces are shown. Quantitated data are expressed as mean \pm SEM. Statistical analysis was performed using a Student's $t$ test and $p$ values of $<0.05$ were considered statistically significant.

\section{Results}

\section{Bok deletion does not alter $\mathrm{IP}_{3} \mathrm{R}$-dependent $\mathrm{Ca}^{2+}$ signaling}

We have shown previously that Bok binds strongly to $\mathrm{IP}_{3} \mathrm{Rs}$ and that essentially all cellular Bok is $\mathrm{IP}_{3} \mathrm{R}$ bound $[17,18]$. To assess stoichiometry, $\mathrm{IP}_{3} \mathrm{R} 1$-Bok complex was immunopurified from $\alpha \mathrm{T} 3$ cells, as described [18], and was subjected to mass spectral analysis. The number of unique Bok and $\mathrm{IP}_{3} \mathrm{R} 1$ peptide spectrum matches obtained was 36 and 329, respectively (an index of relative abundance), and divided by the Bok and $\mathrm{IP}_{3} \mathrm{R} 1$ molecular weights (23.4 and $313 \mathrm{kDa}$, respectively) yielded a Bok to $\mathrm{IP}_{3} \mathrm{R} 1$ ratio of $1.5: 1$. Thus, it is probable that most, if not all of the four, $\mathrm{IP}_{3} \mathrm{R} 1$ polypeptides in a tetrameric channel are bound to Bok. To determine if this $\mathrm{IP}_{3} \mathrm{R}$-bound Bok regulates $\mathrm{IP}_{3} \mathrm{R}$ activity, we deleted Bok in MEFs via CRISPR/Cas9mediated gene editing (Fig. 1a) and measured $\mathrm{IP}_{3} \mathrm{R}$ mediated $\mathrm{Ca}^{2+}$ mobilization (Fig. 1b, c). Bok deletion was specific, as the levels of other pertinent proteins $\left(\mathrm{IP}_{3} \mathrm{Rs}\right.$ and various Bcl-2 family members) were unchanged (Fig. 1a). Examination of $\left[\mathrm{Ca}^{2+}\right]_{\mathrm{c}}$ showed that LPA, which stimulates $\mathrm{IP}_{3}$ formation in fibroblasts [44], induced essentially identical responses in wild-type (WT) and Bok-KO MEFs, in both cell suspension and single-cell analyses (Fig. 1b and Fig. 1c, respectively). Thus, Bok does not appear to regulate $\mathrm{IP}_{3} \mathrm{R}$-mediated $\mathrm{Ca}^{2+}$ mobilization in intact cells.

Due to the close proximity and interaction sites between the ER and mitochondria, $\mathrm{IP}_{3} \mathrm{R}$-mediated $\mathrm{Ca}^{2+}$ mobilization from the ER results in $\mathrm{Ca}^{2+}$ transfer to the mitochondrial matrix [45-47]. To determine if Bok deletion altered mitochondrial $\mathrm{Ca}^{2+}$ levels, $\left[\mathrm{Ca}^{2+}\right]_{\mathrm{m}}$ was measured using $2 \mathrm{mtD} 3 \mathrm{cpv}$, a FRET-based indicator [42]. However, LPA caused an identical $\left[\mathrm{Ca}^{2+}\right]_{\mathrm{m}}$ increase in control and Bok-KO MEFs (Fig. 1d), indicating that Bok does not regulate $\mathrm{Ca}^{2+}$ transfer to the mitochondrial matrix.

We have shown previously that Bok binds preferentially to $\mathrm{IP}_{3} \mathrm{R} 1$ and $\mathrm{IP}_{3} \mathrm{R} 2$, but not $\mathrm{IP}_{3} \mathrm{R} 3$ [18], and since MEFs express all $\mathrm{IP}_{3} \mathrm{R}$ types (Fig. 1a), it is possible that unregulated $\mathrm{IP}_{3} \mathrm{R} 3$ could be masking the effect of Bok on $\mathrm{IP}_{3} \mathrm{R}$ activity. Thus, to directly assess $\mathrm{IP}_{3} \mathrm{R} 1$ activity we examined $\alpha \mathrm{T} 3$ cells, the cell type in which we originally discovered that endogenous Bok and $\mathrm{IP}_{3} \mathrm{R} 1$ interact strongly [18], and in which $\mathrm{IP}_{3} \mathrm{R} 1$ constitutes $\sim 99 \%$ of total $\mathrm{IP}_{3} \mathrm{R}$ content [37]. Bok was specifically deleted in $\alpha \mathrm{T} 3$ cells via CRISPR/Cas9-mediated gene editing (Fig. 1e) and $\left[\mathrm{Ca}^{2}\right.$ $\left.{ }^{+}\right]_{\mathrm{c}}$ was measured (Fig. 1f). Examination of $\left[\mathrm{Ca}^{2+}\right]_{\mathrm{c}}$ showed that $\mathrm{GnRH}$, which stimulates $\mathrm{IP}_{3}$ formation in $\alpha \mathrm{T} 3$ cells [48], induced essentially identical responses in WT and Bok-KO $\alpha \mathrm{T} 3$ cells (Fig. 1f). Thus, Bok does not appear to regulate $\mathrm{IP}_{3} \mathrm{R} 1$-mediated $\mathrm{Ca}^{2+}$ mobilization.

Since feedback regulation of the various proteins that regulate $\left[\mathrm{Ca}^{2+}\right]_{\mathrm{c}}$ [20] could potentially account for global increases in $\left[\mathrm{Ca}^{2+}\right]_{c}$ being unaltered in the Bok-KO cell lines, we also directly measured $\mathrm{IP}_{3} \mathrm{R} 1$ channel activity using the on-nuclear patch-clamp technique applied to $\mathrm{IP}_{3} \mathrm{R} 1-3 \mathrm{KO}$ DT40 chicken $\mathrm{B}$ cells reconstituted with rat $\mathrm{IP}_{3} \mathrm{R} 1$ (DT40-3KO $+\mathrm{rIP}_{3} \mathrm{R} 1$ cells) [43] (Fig. 1g, lane 3). Bok binds strongly to both endogenous and exogenous $\mathrm{IP}_{3} \mathrm{R} 1$ (Fig. 1g, lanes 1 and 3, respectively), but the absence of $\mathrm{IP}_{3} \mathrm{Rs}$ in DT40-3KO cells caused a dramatic decline in Bok levels (Fig. 1g, lane 2), similar to that seen previously in $\mathrm{IP}_{3} \mathrm{R} 1-\mathrm{KO} \alpha \mathrm{T} 3$ cells [17]. Bok was specifically deleted from DT40-3KO + rIP $\mathrm{IP}_{3} 1$ cells via CRISPR/Cas9-mediated gene editing (Fig. 1h), and single-channel recordings of $\mathrm{rIP}_{3} \mathrm{R} 1$ produced similar traces in control and Bok-KO cells (Fig. 1i). Further, quantification of the $\mathrm{IP}_{3} \mathrm{R} 1$ channel activity showed that Bok deletion did not alter channel open probability $\left(P_{0}\right)$ (Fig. $1 \mathrm{j}$ ), burst lengths, or interburst intervals (data not shown). Overall, the data in Fig. 1 show that despite Bok having a strong interaction with $\mathrm{IP}_{3} \mathrm{Rs}$, it does not appear to regulate their channel activity.

\section{Bok deletion and depletion results in mitochondrial fragmentation}

While measuring $\left[\mathrm{Ca}^{2+}\right]_{\mathrm{m}}$ with $2 \mathrm{mtD} 3 \mathrm{cpv}$ (Fig. 1d), we noticed that the mitochondria in Bok-KO MEFs were morphologically different from control MEFs (data not shown). To better define this difference, mitochondria were labeled with DsRed2-Mito, a fluorescent protein that labels mitochondria specifically, via transient transfection and high-resolution images were taken using SIM. While mitochondria in control MEFs displayed the typical elongated morphology [29], mitochondria in Bok-KO MEFs were more fragmented (Fig. 2a). Quantification of images showed that Bok KO resulted in significantly more mitochondrial particles per cell, a decrease in particle volume, and an increase in particle sphericity (Fig. 2b-d, respectively). To determine if acute depletion of Bok has a similar effect to long-term CRISPR/Cas9-mediated Bok KO, shRNA was used to acutely "knockdown" (KD) Bok 
A

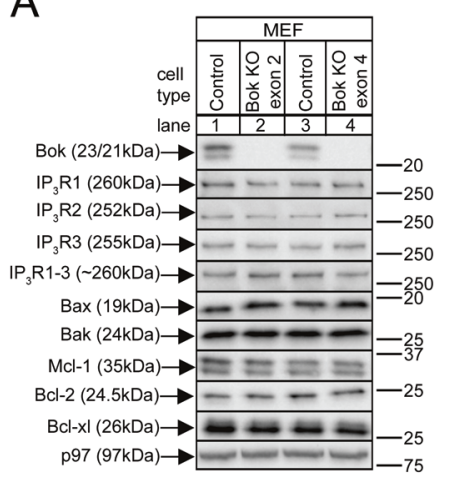

$\mathrm{D}$

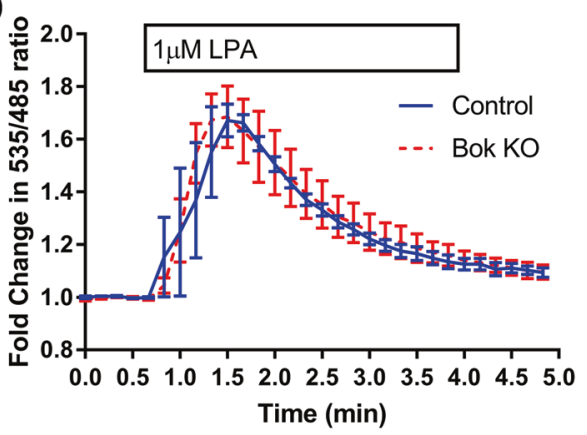

B

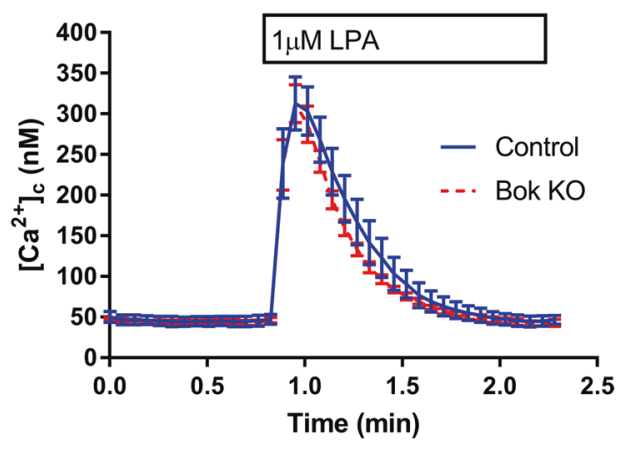

$E$

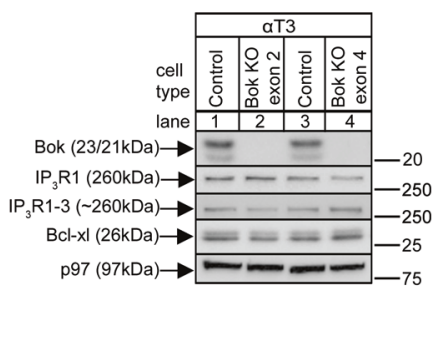

C

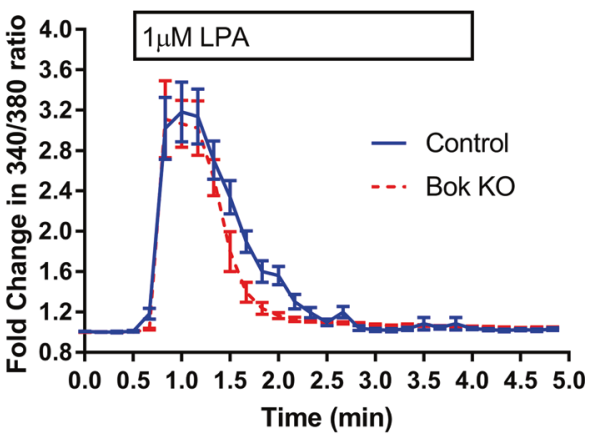

$\mathrm{F}$
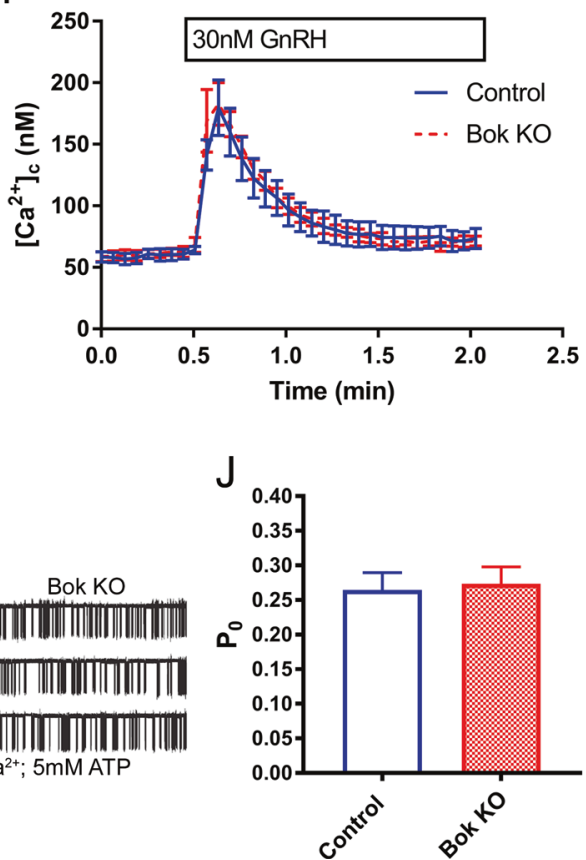

Fig. 1 Bok (Bcl-2-related ovarian killer) deletion does not alter calcium mobilization via inositol 1,4,5-trisphosphate receptors $\left(\mathrm{IP}_{3} \mathrm{Rs}\right)$ or calcium influx into mitochondria. a Levels of Bok and other pertinent proteins in lysates from control and Bok-knockout (KO) mouse embryonic fibroblasts (MEFs) obtained by targeting exon 2 (lane 2) or exon 4 (lane 4), with p97 serving as a loading control. b MEFs in suspension were loaded with Fura 2-AM, exposed to $1 \mu \mathrm{M}$ oleoyl-1- $\alpha$ lysophosphatidic acid (LPA) (box), and cytosolic-free $\mathrm{Ca}^{2+}$ concentration $\left(\left[\mathrm{Ca}^{2+}\right]_{\mathrm{c}}\right)$ was calculated from the $340 / 380 \mathrm{~nm}$ fluorescence ratio. EGTA $(3 \mathrm{mM})$ was added $1 \mathrm{~min}$ prior to LPA to reduce extracellular $\left[\mathrm{Ca}^{2+}\right]$ to $\sim 100 \mathrm{nM}[51]$ and eliminate $\mathrm{Ca}^{2+}$ entry. Traces shown are mean \pm SEM; $N=6$. c MEFs on coverslips were loaded with Fura 2-AM, exposed to $1 \mu$ M LPA (box) and the $340 / 380 \mathrm{~nm}$ fluorescence ratio was measured in single cells. Data are expressed as the fold-change in ratio normalized to baseline values. Traces shown are mean \pm SEM; $n=10$ control cells, $n=7$ Bok-KO cells. d MEFs transfected with $2 \mathrm{mtD} 3 \mathrm{cpv}$ on coverslips were exposed to $1 \mu \mathrm{M} \mathrm{LPA}$ (box) and the $535 / 485 \mathrm{~nm}$ fluorescence ratio was measured in single

expression in MEFs (Fig. 2e). Confocal images of mitochondria stained with MitoTracker showed that control MEFs displayed the typical elongated mitochondrial cells. Data are expressed as the fold-change in ratio normalized to baseline values. Traces shown are mean \pm SEM; $n=104$ control cells, $n=134$ Bok-KO cells. e Levels of Bok and other pertinent proteins in lysates from $\alpha \mathrm{T} 3$ control and Bok-KO cells obtained by targeting exon 2 (lane 2) or exon 4 (lane 4), with p97 serving as a loading control. f $\alpha \mathrm{T} 3$ cells in suspension were loaded with Fura 2-AM, exposed to 30 $\mathrm{nM}$ gonadotropin-releasing hormone (GnRH) (box), and $\left[\mathrm{Ca}^{2+}\right]_{\mathrm{c}}$ was calculated from the $340 / 380 \mathrm{~nm}$ fluorescence ratio. Traces shown are mean $\pm \mathrm{SEM} ; N=5$. $\mathrm{g}$ Lysates and anti-IP ${ }_{3} \mathrm{R} 1$ immunoprecipitations (IPs) from various DT40 cell lines. h Levels of Bok and other pertinent proteins in lysates from DT40-3KO $+\mathrm{rIP}_{3} \mathrm{R} 1$ control and Bok-KO cells obtained by targeting exon 4 , with p97 serving as a loading control. i Single-channel recordings of $\mathrm{rIP}_{3} \mathrm{R} 1$ activity upon stimulation by $1 \mu \mathrm{M}$ $\mathrm{IP}_{3}, 5 \mathrm{mM}$ ATP, and $200 \mathrm{nM} \mathrm{Ca}^{2+}$ in nuclear patches from DT40$3 \mathrm{KO}+\mathrm{rIP}_{3} \mathrm{R} 1$ control and exon 4 Bok-KO cells; $N=3$. j Histogram of the open probability $\left(P_{0}\right)$ for $\mathrm{rIP}_{3} \mathrm{R} 1$ in control and exon 4 Bok-KO cells; mean \pm SEM, $N=3$

morphology, but mitochondria in Bok-KD MEFs were more fragmented, similar to Bok-KO cells (Fig. 2f). Quantification of images showed that Bok KD resulted in 

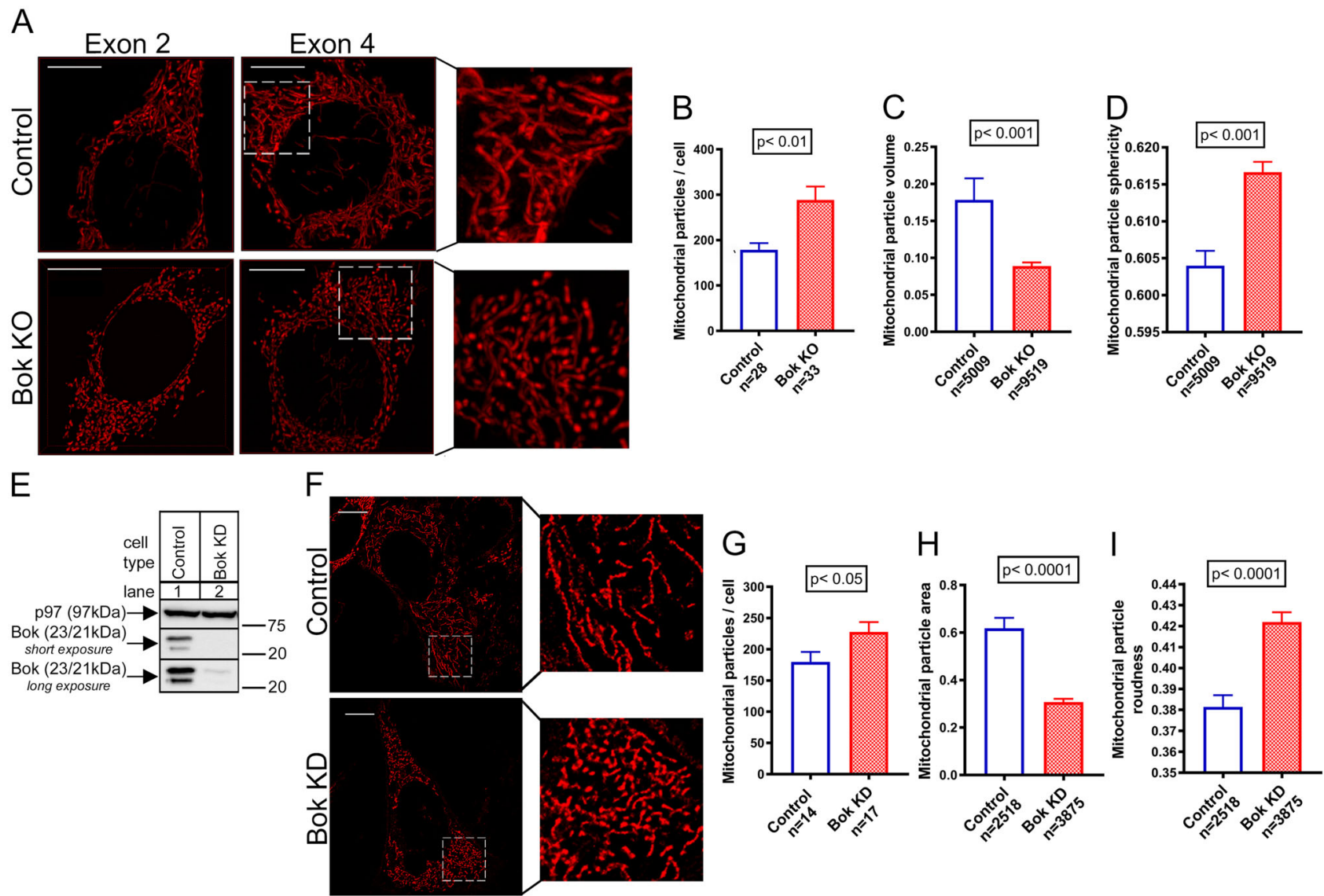

Fig. 2 Bok (Bcl-2-related ovarian killer) deletion and acute depletion causes mitochondrial fragmentation. a Representative confocal images of two independently derived control and Bok-KO mouse embryonic fibroblast (MEF) cell lines (either exon 2 or exon 4 targeted), transiently expressing DsRed2-Mito, and captured using a structured illumination microscope (SIM) (scale bar $=5 \mu \mathrm{m})$. The white boxed regions are further magnified in the expanded images (right side). b-d Quantified measurements of mitochondrial particle number/cell, particle volume, and particle sphericity in control and Bok-KO MEFs. Histograms are mean \pm SEM, with $p$ values comparing control to BokKO cells via unpaired Student's $t$ test, and $n$ designating cell number

significantly more mitochondrial particles per cell, a decrease in particle area, and an increase in particle roundness (Fig. 2g-i, respectively). Thus, both long-term deletion and acute depletion of Bok causes mitochondrial fragmentation.

\section{Exogenous Bok normalizes mitochondrial morphology}

To confirm that the mitochondrial fragmentation seen upon Bok deletion or depletion (Fig. 2) is specifically due to loss of Bok and not possibly caused by off-target effects of genome editing, exogenous Bok (Bok tagged at the $\mathrm{N}$ terminus with the triple FLAG epitope; 3F-WTBok) was stably expressed in Bok-KO MEFs at levels similar to endogenous Bok (Fig. 3a). Note that exogenous 3F- or mitochondrial particle number. e Levels of Bok in lysates from control and Bok-KD MEFs, with p97 serving as a loading control. f Representative confocal images of control and Bok-KD MEFs loaded with MitoTracker Red (scale bar $=10 \mu \mathrm{m}$ ). The white boxed regions are further magnified in the expanded images (right side). $\mathbf{g}-\mathbf{i}$ Quantified measurements of mitochondrial particle number/cell, particle area, and particle roundness in control and Bok-KD MEFs. Histograms are mean \pm SEM, with $p$ values comparing control to Bok-KD cells via unpaired Student's $t$ test, and $n$ designating cell number or mitochondrial particle number

WTBok, like endogenous Bok, expresses as multiple species due to alternative translation initiation [17]. Similar to endogenous Bok, exogenous 3F-WTBok interacts strongly with $\mathrm{IP}_{3} \mathrm{R} 1$, as indicated by co-IP (Fig. 3b, lanes 1 and 3, respectively). To assess mitochondrial morphology, mitochondria were labeled with DsRed2-Mito via transient transfection. Bok-KO ${ }^{+ \text {WTBok }}$ MEFs displayed the typical elongated mitochondrial morphology seen in control MEFs, rather than the fragmented morphology seen in Bok-KO MEFs (Fig. 3c), and quantification of images showed that reconstitution of Bok expression with 3F-WTBok restored mitochondrial morphology parameters to the levels observed in control cells (Fig. 3d-f). Thus, the mitochondrial fragmentation seen upon Bok deletion or depletion is specifically due to the loss of Bok. 


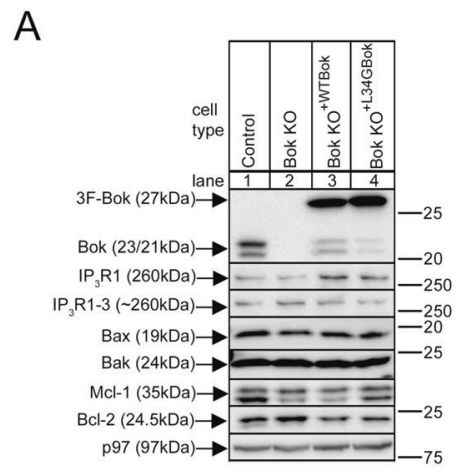

B

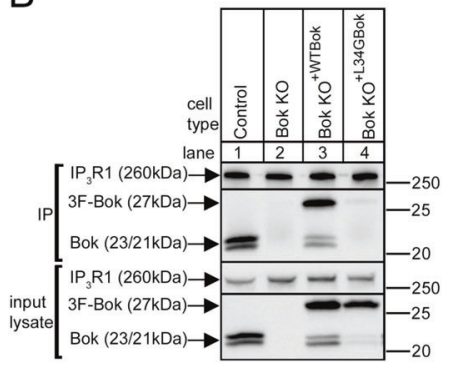

C
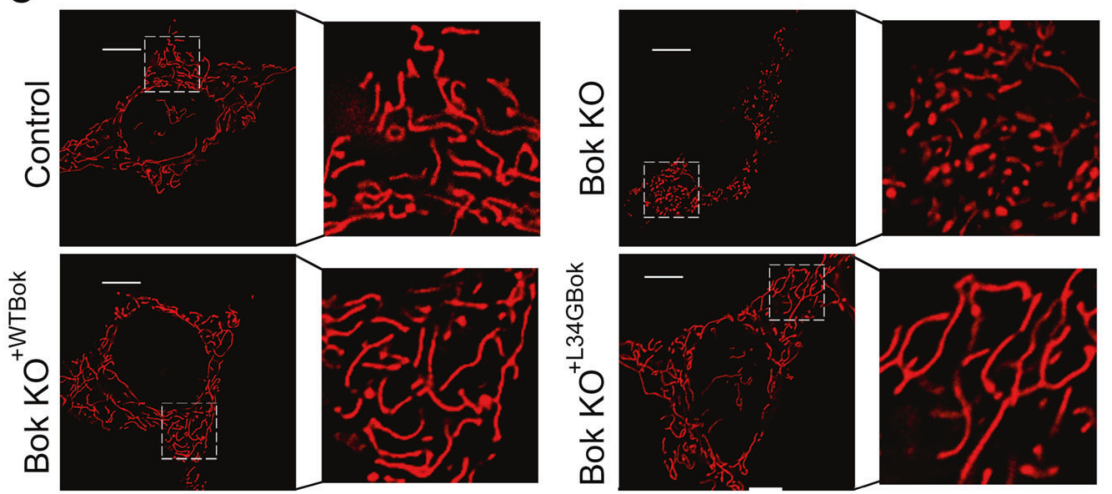

$\mathrm{E}$
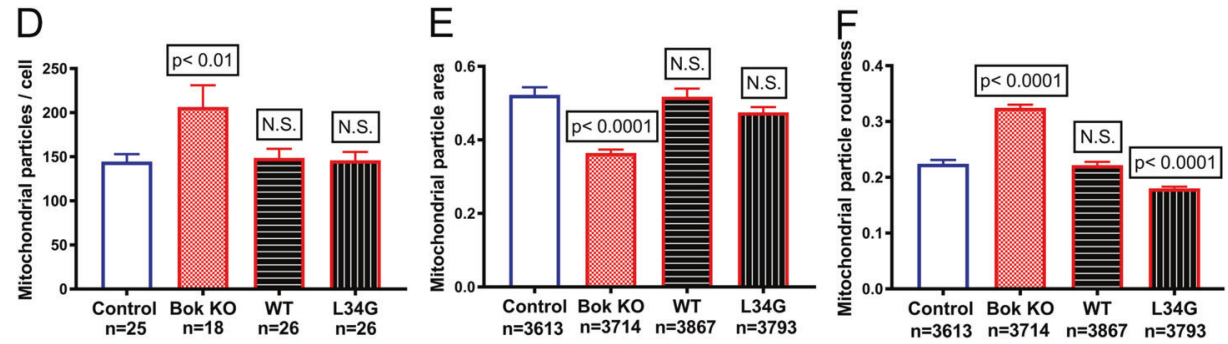

Fig. 3 Exogenous Bok (Bcl-2-related ovarian killer) reverses mitochondrial fragmentation in Bok-KO mouse embryonic fibroblasts (MEFs). a Levels of Bok and other pertinent proteins in lysates from control and Bok-KO MEFs and Bok-KO cell lines stably expressing 3F-WTBok (Bok $\mathrm{KO}^{+ \text {WTBok}}$, lane 3) or 3F-L34GBok (Bok KO +L34GBok, lane 4), with p97 serving as a loading control. b Anti-IP ${ }_{3} \mathrm{R} 1$ immunoprecipitations (IPs) and input cell lysates were probed in immunoblots for the proteins indicated. c Representative confocal images of control, Bok-KO, Bok-KO ${ }^{+ \text {WTBok }}$, Bok-KO ${ }^{+\mathrm{L} 34 \mathrm{GBok}} \mathrm{MEF}$

To determine whether the maintenance of normal mitochondrial morphology by Bok requires the Bok- $P_{3} \mathrm{R} 1$ interaction, 3F-L34GBok, an $\mathrm{IP}_{3} \mathrm{R} 1$ binding-deficient Bok mutant [17], was also stably expressed in Bok-KO MEFs (Fig. 3a, lane 4). Unlike 3F-WTBok, 3F-L34GBok does not co-IP with $\mathrm{IP}_{3} \mathrm{R} 1$ (Fig. 3b, lane 4). Interestingly, 3FL34GBok also normalized mitochondrial morphology parameters to the levels observed in control cells (Fig. 3c-f), and actually enhanced mitochondrial elongation, as indicated by a decrease in mitochondrial particle roundness (Fig. 3f). Thus, maintenance of normal mitochondrial morphology does not require that Bok be $\mathrm{IP}_{3} \mathrm{R}$ bound, but rather merely requires that Bok be present in the ER membrane.

\section{Bok deletion reduces mitochondrial fusion rate and motility}

To begin to examine why Bok deletion or depletion causes mitochondrial fragmentation, mitochondrial fusion rate was measured by assessing mito-PAGFP mobility [30], since other Bcl-2 protein family members (i.e., Bak and Bax) have been reported to regulate this process [29]. After photoactivation, GFP signal spread rapidly throughout the mitochondrial network in control MEFs (Fig. 4a), indicative cell lines transiently expressing DsRed2-Mito (scale bar $=10 \mu \mathrm{m}$ ). Further magnified regions are shown in expanded white boxes (right side). d-f Quantified measurements of mitochondrial particle number/ cell, particle area, and particle roundness in control, Bok-KO, Bok-KO +WTBok and Bok-KO ${ }^{+\mathrm{L} 34 \mathrm{GBok}}$ MEFs. Histograms are mean \pm SEM, with $p$ values comparing control to Bok-KO, Bok-KO ${ }^{+ \text {WTBok }}$, and Bok-KO ${ }^{+\mathrm{L} 34 \mathrm{GBok}}$ via unpaired Student's $t$ test, and $n$ designating the cell number or mitochondrial particle number. $P$ values $>0.05$ were considered not significant (NS)

of efficient mitochondrial fusion [30]. In contrast, little to no spread was seen in Bok-KO MEFs (Fig. 4a), indicative of deficient mitochondrial fusion. Quantification showed that the increase in the fraction of GFP-positive mitochondrial particles from $0 \mathrm{~min}$ to $30 \mathrm{~min}$ was dramatically lowered in the Bok-KO MEFs (Fig. 3b). Thus, Bok KO results in a decrease in mitochondrial fusion rate.

In order to determine if Bok deletion completely blocks or just inhibits mitochondrial fusion, mitochondria were labeled with DsRed2-Mito via transient transfection and treated with Mdivi-1 (a mitochondrial fission inhibitor) [49]. Bok-KO MEFs treated with Mdivi-1 converted the fragmented mitochondria to a more elongated phenotype, typical of that seen in control cells (Fig. 4c-f) and indicating that some fusion must still be occurring. To determine if the decrease in mitochondrial fusion rate could be due to a change in mitochondrial motility, mitochondrial velocity was measured in control and Bok-KO MEFs. Interestingly, Bok deletion significantly reduced mitochondrial velocity (Fig. 4g). Thus, fusion is not blocked, but is significantly reduced in Bok-KO MEFs, perhaps because of a decrease in mitochondrial motility.

The decrease in mitochondrial fusion rate and mitochondrial fragmentation seen in Bok-KO MEFs could be due to 

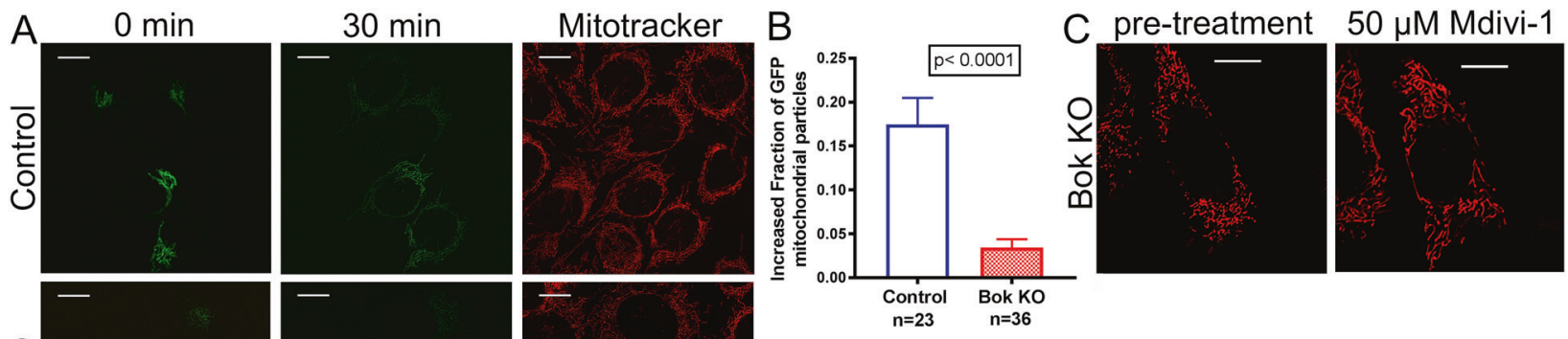
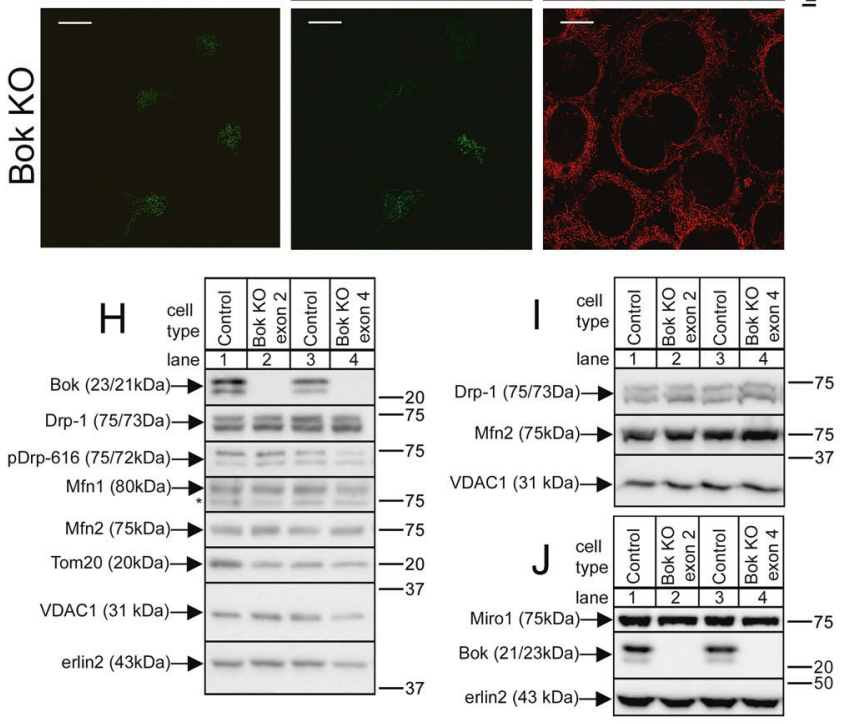

Fig. 4 Bok (Bcl-2-related ovarian killer) deletion decreases mitochondrial fusion rate and velocity without altering the levels of fusion/ fission and Miro proteins. a Representative confocal images of control and Bok-knockout (KO) mouse embryonic fibroblasts (MEFs) transiently expressing mito-PAGFP and stained with MitoTracker Deep Red, followed by photoactivation of one region of interest per cell. Cells were imaged immediately after photoactivation and $30 \mathrm{~min}$ later (scale bar $=10 \mu \mathrm{m}$ ). b Quantification of the increased fraction of green fluorescent protein (GFP)-positive mitochondria from 0 to 30 min. Histograms are mean \pm SEM, with $p$ values comparing control to Bok-KO cells via unpaired Student's $t$ test, and $n$ designating cell number. c Representative confocal image of Bok-KO MEFs transiently expressing DsRed2-Mito, pre-treatment and post-treatment with $50 \mu \mathrm{M}$ Mdivi-1 for $1 \mathrm{~h}$ (scale bar $=10 \mu \mathrm{m})$. d-f Quantified measurements of mitochondrial particle number/cell, particle area, and

changes in the levels of proteins that regulate mitochondrial fusion or fission. However, this was not the case as the levels of Mfn1 and Mfn2 (fusion mediators) and Drp-1 (fission mediator) in cell lysates were unaltered by Bok KO (Fig. 4h). Phosphorylation of Drp-1 at amino acid 616 induces mitochondrial fission activity [27], but pDrp-616 levels in cell lysates were also unaltered by Bok KO (Fig. 4h). Finally, Drp-1 translocation from the cytosol to mitochondria regulates fission [27], but Drp-1 levels in crude mitochondrial fractions were unaltered by Bok KO (Fig. 4i). Likewise, the level of Miro1, the mitochondrial GTPase that sustains mitochondrial motility $[25,27]$, was unaltered by Bok KO (Fig. 4j). Thus, the decreases in mitochondrial fusion rate and
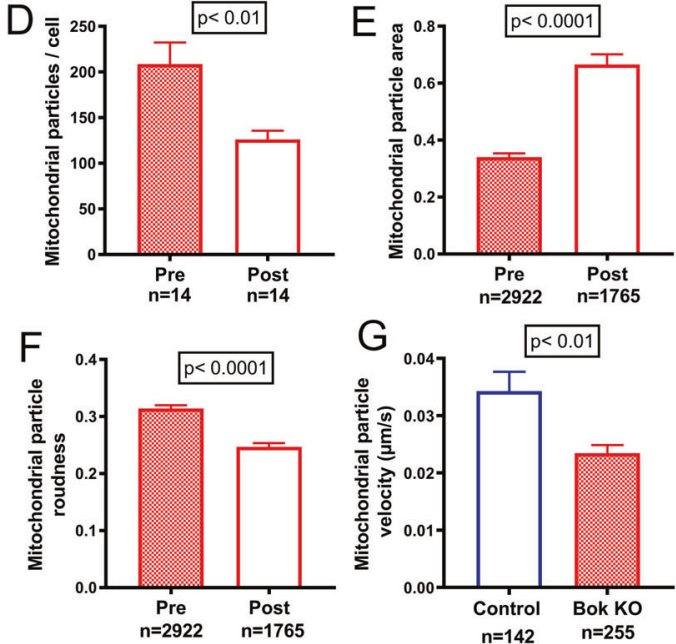

particle roundness in Bok-KO MEFs pre- and post-treatment. Histograms are mean \pm SEM, with $p$ values comparing Bok-KO MEFs pretreatment to post-treatment via unpaired Student's $t$ test, and $n$ designating cell number or mitochondrial particle number. $\mathbf{g}$ Quantified measurements of mitochondrial particle velocity in control and BokKO MEFs transiently expressing DsRed2-Mito. Histograms are mean $\pm \mathrm{SEM}$, with $p$ values comparing control to Bok-KO MEFs via unpaired Student's $t$ test, and $n$ designating the number of mitochondrial particles measured in 12 cells. h Levels of fission and fusion mediators and other pertinent proteins in lysates from control and BokKO MEFs, with erlin2 serving as a loading control. i Levels of fission and fusion mediators in crude mitochondrial fractions. $\mathbf{j}$ Levels of Miro1 and other pertinent proteins in lysates from control and Bok-KO MEFs, with erlin2 serving as a loading control

velocity seen in Bok-KO MEFs are not due to changes in the levels of proteins that regulate mitochondrial dynamics.

\section{Bok deletion does not inhibit mitochondrial bioenergetics or cell growth}

Since Bok KO results in mitochondrial fragmentation, we sought to determine if this affected mitochondrial bioenergetics. Mitochondrial respiration was analyzed in control and Bok-KO MEFs (Fig. 5a), before and after the addition of various electron transport chain (ETC) complex inhibitors; oligomycin (complex $\mathrm{V}$ inhibitor) to determine the amount of ATP production linked to respiration, FCCP 

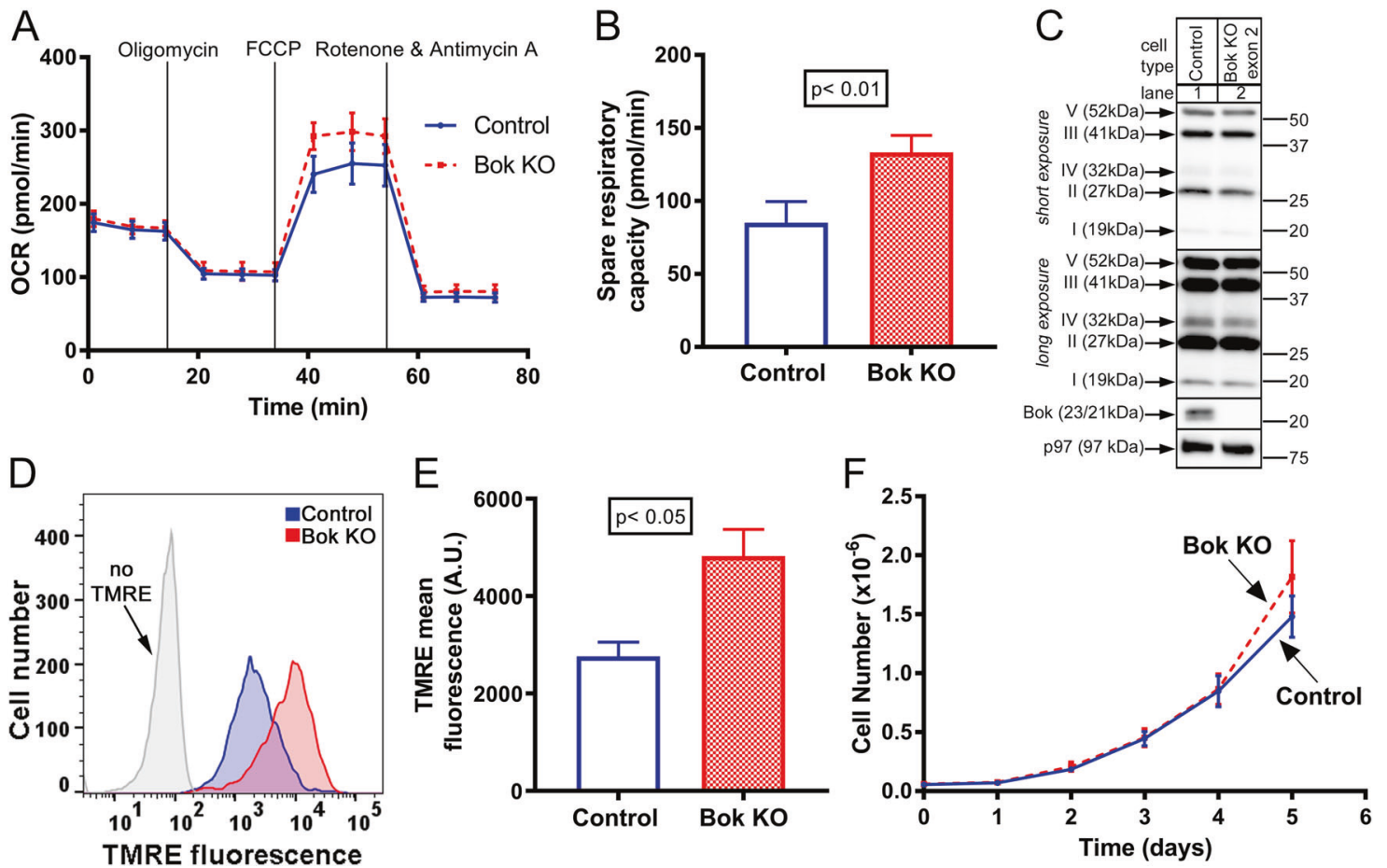

Fig. 5 Bok (Bcl-2-related ovarian killer) deletion increases mitochondrial spare respiratory capacity and membrane potential, but has no effect on cell growth. a Oxygen consumption rate (OCR) in control and Bok-knockout (KO) mouse embryonic fibroblasts (MEFs) treated with oligomycin, carbonyl cyanide- $p$-trifluoromethoxy-phenyl-hydrazone (FCCP), and a combination of rotenone and antimycin A (traces are mean $\pm \mathrm{SEM}, N=4)$. b Spare respiratory capacity in control and Bok-KO MEFs. Histograms are mean \pm SEM, $N=4$, with $p$ values comparing control to Bok KO via unpaired Student's $t$ test. $\mathbf{c}$ Levels of

(ionophore that collapses the inner membrane gradient) to cause the ETC to function at its maximal rate, and a combination of rotenone and antimycin A (complex I and III inhibitor, respectively) to shut down the ETC and determine non-mitochondrial respiration. Control and Bok-KO MEFs displayed a similar bioenergetic profile (Fig. 5a), but, interestingly, Bok-KO MEFs had significantly higher spare respiratory capacity (difference between maximal and basal OCR) (Fig. 5b), despite the levels of ETC protein complexes being unaltered by Bok KO (Fig. 5c). Surprisingly, Bok-KO MEFs also exhibited a significantly higher mitochondrial membrane potential, as indicated by enhanced TMRE fluorescence (Fig. 5d, e). Thus, the mitochondrial fragmentation seen in Bok-KO MEFs does not inhibit mitochondrial bioenergetics, and in fact, correlates with enhanced spare respiratory capacity and membrane potential. Consistent with this lack of inhibition of bioenergetics, cellular proliferation was not impaired (Fig. 5f).

\section{Bok deletion does not affect apoptosis}

Since Bok is a Bcl-2 family member, we sought to determine if endogenous Bok contributes to apoptotic signaling by electron transport chain (ETC) complexes in lysates from control and Bok-KO MEFs, with p97 serving as a loading control. d Representative histogram of control and Bok-KO MEFs incubated with 500 $\mathrm{nM}$ tetramethylrhodamine ethyl ester perchlorate (TMRE) and analyzed by flow cytometry. Unstained control cells are also shown for comparison. e Mean TMRE fluorescence in control and Bok-KO MEFs. Histograms are mean \pm SEM, $N=4$, with $p$ values comparing control to Bok KO via unpaired Student's $t$ test. f Cell growth curves from control and Bok-KO MEFs (mean $\pm \mathrm{SEM} ; N=5$ )

exposing control and Bok-KO MEFs to various apoptotic stimuli; SST (a non-selective protein kinase inhibitor), MG132 (a proteasome inhibitor), TG (a sarco/ER $\mathrm{Ca}^{2+}$ ATPase pump inhibitor), BFA (a Golgi/ER transport inhibitor), and TN (a N-linked glycosylation inhibitor). However, Fig. 6 (lanes 3-12) shows that regardless of the stimulus, control and Bok-KO MEFs displayed very similar levels of apoptotic activity as assessed by PARP and caspase- 3 cleavage $(\mathrm{cC} 3)$, and quantification of $\mathrm{cC} 3$ showed no significant changes $(p>0.05$, as determined by a unpaired Student's $t$ test) (Fig. 6). Thus, despite Bok being a Bcl-2 protein family member, endogenous Bok does not seem to play a significant role in stimulus-induced apoptosis.

\section{Discussion}

In summary, we find that (i) despite Bok having a strong interaction with $\mathrm{IP}_{3} \mathrm{Rs}$, it does not regulate $\mathrm{IP}_{3} \mathrm{R}$ channel activity, (ii) both long-term deletion and acute depletion of Bok results in mitochondrial fragmentation, (iii) maintenance of normal mitochondrial morphology is dependent on the presence of Bok, but not the Bok-IP ${ }_{3} \mathrm{R}$ interaction, (iv) the 


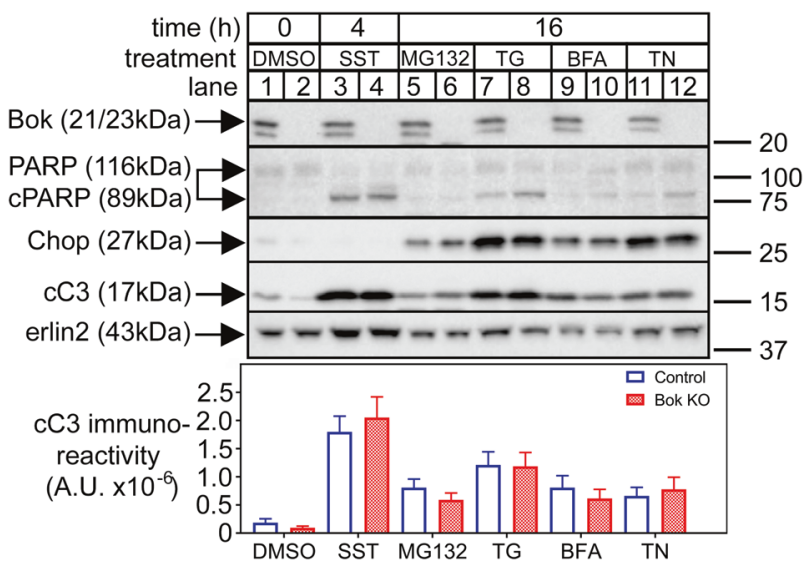

Fig. 6 Bok (Bcl-2-related ovarian killer) deletion does not alter apoptotic responses. Control and Bok-knockout (KO) mouse embryonic fibroblasts (MEFs) (odd and even numbered lanes, respectively) were incubated as indicated with dimethyl sulfoxide (DMSO), $1 \mu \mathrm{M}$ staurosporine (SST), $10 \mu \mathrm{M}$ MG132, $1 \mu \mathrm{M}$ thapsigargin (TG), $0.5 \mu \mathrm{g} / \mathrm{mL}$ brefeldin A (BFA), or $0.5 \mu \mathrm{g} / \mathrm{mL}$ tunicamycin (TN), and cell lysates were subjected to sodium dodecyl sulfatepolyacrylamide gel electrophoresis (SDS-PAGE) and probed in immunoblots for the proteins indicated, with erlin2 serving as a loading control. The histogram shows quantitated cleaved caspase-3 (cC3) immunoreactivity from multiple independent control and BokKO MEF cell lines $(N=5)$

mitochondrial fragmentation caused by Bok deletion is due to a decrease in mitochondrial fusion rate, perhaps because of a decrease in motility, (v) Bok deletion enhances mitochondrial bioenergetics, and, lastly, (vi) despite Bok being a Bcl-2 family member, it does not play a significant role in stimulusinduced apoptosis. Overall, these data reveal a novel cellular role for Bok in maintaining mitochondrial morphology by sustaining mitochondrial fusion.

We reported previously that control MEFs and Bok-KO MEFs derived from Bok-KO mice had identical $\mathrm{IP}_{3} \mathrm{R}$ mediated $\mathrm{Ca}^{2+}$ responses [18]. However, a caveat was that $\mathrm{IP}_{3} \mathrm{R}$ expression was altered by Bok $\mathrm{KO}$, with $\mathrm{IP}_{3} \mathrm{R} 1$ levels increased and $\mathrm{IP}_{3} \mathrm{R} 2$ and $\mathrm{IP}_{3} \mathrm{R} 3$ levels decreased, possibly due to adaptation to the long-term germline loss of Bok [18]. In contrast, the Bok-KO MEFs generated in the present study using CRISPR/Cas9-mediated gene editing did not have altered $\mathrm{IP}_{3} \mathrm{R}$ expression (Fig. 1a). Despite the thorough investigation of these MEFs and other Bok-KO cells shown in Fig. 1, Bok was not seen to regulate $\mathrm{IP}_{3} \mathrm{R}$-induced $\mathrm{Ca}^{2+}$ mobilization in intact cells or $\mathrm{IP}_{3} \mathrm{R}$ channel activity. This is surprising for several reasons: (i) Bok binds strongly to $\mathrm{IP}_{3} \mathrm{Rs}$, (ii) essentially all cellular Bok is $\mathrm{IP}_{3} \mathrm{R}$ bound $[17,18]$, (iii) each of the four polypeptides in an $\mathrm{IP}_{3} \mathrm{R}$ tetramer appear to be Bok bound, and (iv) Bok binds much more efficiently to $\mathrm{IP}_{3} \mathrm{Rs}$ than other $\mathrm{Bcl}-2$ protein family members (e.g., Bcl-2 and $\mathrm{Bcl}-\mathrm{xl})$ [18] that have been reported to regulate $\mathrm{IP}_{3} \mathrm{Rs}$ [24]. Further, Bok KO did not alter $\left[\mathrm{Ca}^{2+}\right]_{\mathrm{m}}$, indicating that $\mathrm{Ca}^{2+}$ transfer to mitochondria is not regulated by Bok and suggesting that ER-mitochondria contact sites are normal in Bok-KO cells. It remains a possibility, however, that Bok could regulate $\mathrm{IP}_{3} \mathrm{R}$-independent $\mathrm{Ca}^{2+}$ homeostasis at the ER (e.g., $\mathrm{Ca}^{2+}$ uptake or passive leak) [50], or that effects of Bok on $\mathrm{IP}_{3} \mathrm{R}$ activity could be revealed under different conditions.

Rather than affecting $\mathrm{IP}_{3} \mathrm{R}$ activity, Bok deletion was found to cause mitochondrial fragmentation by inhibiting mitochondrial fusion. Interestingly, mitochondrial fragmentation is also seen when other Bcl-2 protein family members are deleted (e.g., Bak, Bax, Mcl-1, Bcl-xl, and Bcl-2), apparently due to their interactions with fusion or fission mediators [28-34]. For example, Bak and Bax have been shown to maintain normal mitochondrial morphology by regulating mitochondrial fusion rate via interactions with Mfn2 [28-31]. However, using co-IP we were unable to detect interactions between Bok and Mfn1/2 or Drp-1 (data not shown). This does not rule out the possibility that such interactions occur, since co-IP identifies only strong proteinprotein interactions, and Bok localization to the ER may limit it from interacting strongly with fusion or fission mediators, which predominately localize to the mitochondria [27]. Alternatively, Bok could sustain mitochondrial fusion indirectly by interacting with a $\mathrm{Bcl}-2$ protein family member that in turn has a direct interaction with a fusion or fission mediator. For example, Bok has been reported to interact with Mcl-1 by a yeast two-hybrid system [5] and Mcl-1 interacts with Drp-1 to regulate mitochondrial dynamics [33]. This idea is consistent with the notion that $\mathrm{Bcl}-2$ family members form a network that acts cooperatively to regulate mitochondrial dynamics [25, 26, 30]. Finally, since we found that Bok deletion decreases mitochondrial particle velocity (Fig. 4g), it is possible that this accounts for the reduced fusion rate in Bok-KO MEFs; a decrease in mitochondrial motility will diminish the likelihood of two mitochondria being in close proximity, which is necessary for their fusion [25, 27]. At present, it is unclear why motility is reduced, as the level of Miro1 was unaltered by Bok KO (Fig. 4j).

Interestingly, both exogenous WTBok and L34GBok, an $\mathrm{IP}_{3} \mathrm{R} 1$ binding-deficient Bok mutant, were able to normalize mitochondrial morphology (Fig. 3). Thus, maintenance of a normal mitochondrial morphology is dependent merely on the presence of Bok, rather than the Bok- $\mathrm{IP}_{3} \mathrm{R}$ interaction. Intriguingly, L34GBok caused a more elongated mitochondrial morphology than WTBok, indicating that "free" Bok (that not bound to $\mathrm{IP}_{3} \mathrm{Rs}$ ) may be a more potent promoter of mitochondrial fusion than tethered Bok, and perhaps that the Bok-IP $\mathrm{P}_{3} \mathrm{R}$ interaction restrains Bok activity in order for it to maintain normal mitochondrial dynamics. At the same time, it is important to note that endogenous Bok is profoundly stabilized by binding to $\mathrm{IP}_{3} \mathrm{Rs}$ [18]. Therefore, the interaction between endogenous $\mathrm{IP}_{3} \mathrm{Rs}$ and Bok is critical for maintaining Bok at levels that can sustain normal mitochondrial fusion. 
Mitochondrial fragmentation induced by $\mathrm{KO}$ of $\mathrm{Bcl}-2$ family members can inhibit mitochondrial bioenergetics in some cases, for example, Mcl-1 KO in MEFs [32], but not always, for example, Bax/Bak double KO in MEFs [29]. Interestingly, the mitochondrial fragmentation induced by Bok KO did not inhibit mitochondrial bioenergetics, but, surprisingly, enhanced mitochondrial spare respiratory capacity (Fig. 5b) and membrane potential (Fig. 5d, e). These changes are not due to an increase in the levels of ETC protein complexes and may simply be due to the increase in mitochondrial surface area that will inevitably result from mitochondrial fragmentation. Such an increase in surface area could enhance ETC activity by deconstraining diffusion. Clearly, additional work is needed to resolve these questions.

Since Bok is a Bcl-2 protein family member, many studies have focused on the possible role that it could play in apoptosis. However, this has not led to a clear consensus view, with studies showing both pro- and anti-apoptotic characteristics $[4,7,9,11-16]$. Indeed, a concern with the numerous studies showing that overexpressed exogenous Bok is proapoptotic $[7,9,11,12]$ is that overexpressed Bok will likely saturate the available $\mathrm{IP}_{3} \mathrm{R}$ binding sites and generate "free Bok" that can cause non-physiological deleterious effects and perturbations to the Bcl-2 family network. The Bok-KO approach to defining the role of Bok has also led to conflicting findings; for example, Bok-KO MEFs derived from germline Bok deletion by two independent groups have shown Bok to have either an anti- or pro-apoptotic role in response to ER stress agents [7, 13-15]. In contrast, the CRISPR/Cas9derived Bok-KO MEFs used in the present study reveal that Bok has no role in apoptosis induced by various stimuli, including ER stress agents (Fig. 6). These differences may be due to adaptation to the long-term loss of Bok in the MEFs derived from KO mice, which do not occur in the Bok-KO MEFs derived from CRISPR/Cas9-mediated gene editing. In summary, our data support the idea that Bok plays little or no role in stimulus-induced apoptosis.

Overall, our findings reveal that an important role of Bok is to maintain mitochondrial morphology by sustaining mitochondrial fusion rate. Further research is needed to determine the mechanism by which Bok regulates mitochondrial fusion, motility, and bioenergetics and the proteins with which Bok interacts, beyond $\mathrm{IP}_{3} \mathrm{Rs}$. Another major question that remains unsolved is precisely how the Bcl-2 family network [1-3] collectively regulates mitochondrial dynamics.

Acknowledgements We thank Dr. Nicholas Deakin for support and suggestions regarding microscopy; Dr. Danielle Sliter and Dr. Richard Youle for facilitating mitochondrial fusion rate measurements; Dr. Forrest Wright and Caden Bonzerato for helpful suggestions; Dr. Ebbing De Jong for assistance with mass spectral analysis; Dr. Thomas Kaufmann for providing anti-Bok; Dr. Jan B Parys for providing antiIP3R1-3; and grant support to RJHW from NIH (DK107944 and GM121621) and the Carol M. Baldwin Breast Cancer Research Fund.

\section{Compliance with ethical standards}

Conflict of interest The authors declare that they have no conflict of interest.

Publisher's note: Springer Nature remains neutral with regard to jurisdictional claims in published maps and institutional affiliations.

\section{References}

1. Kale J, Osterlund EJ, Andrews DW. BCL-2 family proteins: changing partners in the dance towards death. Cell Death Differ. 2018;25:65-80.

2. Kalkavan H, Green DR. MOMP, cell suicide as a BCL-2 family business. Cell Death Differ. 2018;25:46-55.

3. Siddiqui WA, Ahad A, Ahsan H. The mystery of BCL2 family: Bcl-2 proteins and apoptosis: an update. Arch Toxicol. 2015;89:289-317.

4. Ke F, Voss A, Kerr JB, O'Reilly LA, Tai L, Echeverry N, et al. BCL-2 family member BOK is widely expressed but its loss has only minimal impact in mice. Cell Death Differ. 2012;19:915-25.

5. Hsu SY, Kaipia A, McGee E, Lomeli M, Hsueh AJ. Bok is a proapoptotic Bcl-2 protein with restricted expression in reproductive tissues and heterodimerizes with selective anti-apoptotic Bcl-2 family members. Proc Natl Acad Sci USA. 1997;94:12401-6.

6. Hsu SY, Hsueh AJ. A splicing variant of the Bcl-2 member Bok with a truncated $\mathrm{BH} 3$ domain induces apoptosis but does not dimerize with antiapoptotic Bcl-2 proteins in vitro. J Biol Chem. 1998;273:30139-46.

7. Echeverry N, Bachmann D, Ke F, Strasser A, Simon HU, Kaufmann T. Intracellular localization of the BCL-2 family member BOK and functional implications. Cell Death Differ. 2013;20:785-99.

8. Schinzel A, Kaufmann T, Borner C. Bcl-2 family members: integrators of survival and death signals in physiology and pathology [corrected]. Biochim Biophys Acta. 2004;1644:95-105.

9. Fernandez-Marrero Y, Bleicken S, Das KK, Bachmann D, Kaufmann T, Garcia-Saez AJ. The membrane activity of BOK involves formation of large, stable toroidal pores and is promoted by cBID. FEBS J. 2017;284:711-24.

10. Einsele-Scholz S, Malmsheimer S, Bertram K, Stehle D, Johanning J, Manz M, et al. Bok is a genuine multi-BH-domain protein that triggers apoptosis in the absence of Bax and Bak. J Cell Sci. 2016;129:3054.

11. Llambi F, Wang YM, Victor B, Yang M, Schneider DM, Gingras $\mathrm{S}$, et al. BOK is a non-canonical BCL-2 family effector of apoptosis regulated by ER-associated degradation. Cell. 2016;165:421-33.

12. Zheng JH, Grace CR, Guibao CD, McNamara DE, Llambi F, Wang YM, et al. Intrinsic Instability of BOK enables membrane permeabilization in apoptosis. Cell Rep. 2018;23:2083-94 e6.

13. Carpio MA, Michaud M, Zhou W, Fisher JK, Walensky LD, Katz SG. BCL-2 family member BOK promotes apoptosis in response to endoplasmic reticulum stress. Proc Natl Acad Sci USA. 2015;112:7201-6.

14. Carpio MA, Michaud M, Zhou W, Fisher JK, Walensky LD, Katz SG. Reply to Fernandez-Marrero et al.: Role of BOK at the intersection of endoplasmic reticulum stress and apoptosis regulation. Proc Natl Acad Sci USA. 2016;113:E494-5.

15. Fernandez-Marrero Y, Ke F, Echeverry N, Bouillet P, Bachmann $\mathrm{D}$, Strasser A, et al. Is BOK required for apoptosis induced by endoplasmic reticulum stress? Proc Natl Acad Sci USA. 2016;113:E492-3. 
16. D'Orsi B, Engel T, Pfeiffer S, Nandi S, Kaufmann T, Henshall DC, et al. Bok is not pro-apoptotic but suppresses poly ADPribose polymerase-dependent cell death pathways and protects against excitotoxic and seizure-induced neuronal injury. J Neurosci. 2016;36:4564-78.

17. Schulman JJ, Wright FA, Han X, Zluhan EJ, Szczesniak LM, Wojcikiewicz RJ. The stability and expression level of Bok are governed by binding to inositol 1,4,5-trisphosphate receptors. J Biol Chem. 2016;291:11820-8.

18. Schulman JJ, Wright FA, Kaufmann T, Wojcikiewicz RJ. The Bcl-2 protein family member Bok binds to the coupling domain of inositol 1,4,5-trisphosphate receptors and protects them from proteolytic cleavage. J Biol Chem. 2013;288:25340-9.

19. Fan G, Baker ML, Wang Z, Baker MR, Sinyagovskiy PA, Chiu $\mathrm{W}$, et al. Gating machinery of InsP3R channels revealed by electron cryomicroscopy. Nature. 2015;527:336-41.

20. Foskett JK, White C, Cheung KH, Mak DO. Inositol trisphosphate receptor $\mathrm{Ca}^{2+}$ release channels. Physiol Rev. 2007;87:593-658.

21. Raffaello A, Mammucari C, Gherardi G, Rizzuto R. Calcium at the center of cell signaling: interplay between endoplasmic reticulum, mitochondria, and lysosomes. Trends Biochem Sci. 2016;41:1035-49.

22. Rizzuto R, Marchi S, Bonora M, Aguiari P, Bononi A, De Stefani $\mathrm{D}$, et al. $\mathrm{Ca}(2+)$ transfer from the ER to mitochondria: when, how and why. Biochim Biophys Acta. 2009;1787:1342-51.

23. Akl $\mathrm{H}$, Bultynck $\mathrm{G}$. Altered $\mathrm{Ca}(2+)$ signaling in cancer cells: proto-oncogenes and tumor suppressors targeting IP3 receptors. Biochim Biophys Acta. 2013;1835:180-93.

24. Distelhorst CW, Bootman MD. Bcl-2 interaction with the inositol 1,4,5-trisphosphate receptor: role in $\mathrm{Ca}(2+)$ signaling and disease. Cell Calcium. 2011;50:234-41.

25. Aouacheria A, Baghdiguian S, Lamb HM, Huska JD, Pineda FJ, Hardwick JM. Connecting mitochondrial dynamics and life-ordeath events via $\mathrm{Bcl}-2$ family proteins. Neurochem Int. 2017;109:141-61.

26. D’Orsi B, Mateyka J, Prehn JHM. Control of mitochondrial physiology and cell death by the Bcl-2 family proteins Bax and Bok. Neurochem Int. 2017;109:162-70.

27. Chan DC. Fusion and fission: interlinked processes critical for mitochondrial health. Annu Rev Genet. 2012;46:265-87.

28. Hoppins S, Edlich F, Cleland MM, Banerjee S, McCaffery JM, Youle RJ, et al. The soluble form of Bax regulates mitochondrial fusion via MFN2 homotypic complexes. Mol Cell. 2011; 41:150-60.

29. Karbowski M, Norris KL, Cleland MM, Jeong SY, Youle RJ. Role of Bax and Bak in mitochondrial morphogenesis. Nature. 2006;443:658-62.

30. Cleland MM, Norris KL, Karbowski M, Wang C, Suen DF, Jiao S, et al. Bcl-2 family interaction with the mitochondrial morphogenesis machinery. Cell Death Differ. 2011;18:235-47.

31. Brooks C, Wei Q, Feng L, Dong G, Tao Y, Mei L, et al. Bak regulates mitochondrial morphology and pathology during apoptosis by interacting with mitofusins. Proc Natl Acad Sci USA. 2007;104:11649-54.

32. Perciavalle RM, Stewart DP, Koss B, Lynch J, Milasta S, Bathina $\mathrm{M}$, et al. Anti-apoptotic MCL-1 localizes to the mitochondrial matrix and couples mitochondrial fusion to respiration. Nat Cell Biol. 2012;14:575-83.

33. Morciano G, Giorgi C, Balestra D, Marchi S, Perrone D, Pinotti M, et al. Mcl-1 involvement in mitochondrial dynamics is associated with apoptotic cell death. Mol Biol Cell. 2016;27:20-34.
34. Berman SB, Chen YB, Qi B, McCaffery JM, Rucker EB 3rd, Goebbels S, et al. Bcl-x L increases mitochondrial fission, fusion, and biomass in neurons. J Cell Biol. 2009;184:707-19.

35. Wojcikiewicz RJ. Type I, II, and III inositol 1,4,5-trisphosphate receptors are unequally susceptible to down-regulation and are expressed in markedly different proportions in different cell types. J Biol Chem. 1995;270:11678-83.

36. Wojcikiewicz RJ, Xu Q, Webster JM, Alzayady K, Gao C. Ubiquitination and proteasomal degradation of endogenous and exogenous inositol 1,4,5-trisphosphate receptors in alpha T3-1 anterior pituitary cells. J Biol Chem. 2003;278:940-7.

37. Pearce MM, Wang Y, Kelley GG, Wojcikiewicz RJ. SPFH2 mediates the endoplasmic reticulum-associated degradation of inositol 1,4,5-trisphosphate receptors and other substrates in mammalian cells. J Biol Chem. 2007;282:20104-15.

38. Bultynck G, Szlufcik K, Kasri NN, Assefa Z, Callewaert G, Missiaen L, et al. Thimerosal stimulates $\mathrm{Ca}^{2+}$ flux through inositol 1,4,5-trisphosphate receptor type 1 , but not type 3, via modulation of an isoform-specific $\mathrm{Ca}^{2+}$-dependent intramolecular interaction. Biochem J. 2004;381(Part 1):87-96.

39. Mali P, Esvelt KM, Church GM. Cas9 as a versatile tool for engineering biology. Nat Methods. 2013;10:957-63.

40. Ran FA, Hsu PD, Wright J, Agarwala V, Scott DA, Zhang F. Genome engineering using the CRISPR-Cas9 system. Nat Protoc. 2013;8:2281-308.

41. Grynkiewicz G, Poenie M, Tsien RY. A new generation of $\mathrm{Ca}^{2+}$ indicators with greatly improved fluorescence properties. J Biol Chem. 1985;260:3440-50.

42. Harbeck MC, Chepurny O, Nikolaev VO, Lohse MJ, Holz GG, Roe MW. Simultaneous optical measurements of cytosolic $\mathrm{Ca}^{2+}$ and cAMP in single cells. Sci STKE. 2006;2006:p16.

43. Wagner LE 2nd, Yule DI. Differential regulation of the $\operatorname{InsP}(3)$ receptor type-1 and -2 single channel properties by $\operatorname{InsP}(3), \mathrm{Ca}(2)$ $(+)$ and ATP. J Physiol. 2012;590:3245-59.

44. Kelley GG, Kaproth-Joslin KA, Reks SE, Smrcka AV, Wojcikiewicz RJ. G-protein-coupled receptor agonists activate endogenous phospholipase Cepsilon and phospholipase Cbeta3 in a temporally distinct manner. J Biol Chem. 2006;281:2639-48.

45. Csordas G, Varnai P, Golenar T, Roy S, Purkins G, Schneider TG, et al. Imaging interorganelle contacts and local calcium dynamics at the ER-mitochondrial interface. Mol Cell. 2010;39:121-32.

46. Hajnoczky G, Robb-Gaspers LD, Seitz MB, Thomas AP. Decoding of cytosolic calcium oscillations in the mitochondria. Cell. 1995;82:415-24.

47. Rizzuto R, Brini M, Murgia M, Pozzan T. Microdomains with high $\mathrm{Ca}^{2+}$ close to IP3-sensitive channels that are sensed by neighboring mitochondria. Science. 1993;262:744-7.

48. Xu Q, Farah M, Webster JM, Wojcikiewicz RJ. Bortezomib rapidly suppresses ubiquitin thiolesterification to ubiquitin-conjugating enzymes and inhibits ubiquitination of histones and type I inositol 1,4,5-trisphosphate receptor. Mol Cancer Ther. 2004;3:1263-9.

49. Cassidy-Stone A, Chipuk JE, Ingerman E, Song C, Yoo C, Kuwana T, et al. Chemical inhibition of the mitochondrial division dynamin reveals its role in Bax/Bak-dependent mitochondrial outer membrane permeabilization. Dev Cell. 2008;14:193-204.

50. Krebs J, Agellon LB, Michalak M. $\mathrm{Ca}(2+)$ homeostasis and endoplasmic reticulum (ER) stress: an integrated view of calcium signaling. Biochem Biophys Res Commun. 2015;460:114-21.

51. Wojcikiewicz RJ, Tobin AB, Nahorski SR. Muscarinic receptormediated inositol 1,4,5-trisphosphate formation in SH-SY5Y neuroblastoma cells is regulated acutely by cytosolic $\mathrm{Ca}^{2+}$ and by rapid desensitization. J Neurochem. 1994;63:177-85. 